\title{
CAPBREU DE LA CARTOIXA. ELS DOMINIS DE SCALA DEI A LA COMARCA DE VALLS
}

Josefina Cubells $i$ Llorens

A l'Arxiu Històric de Tarragona, en un plec de documents dels fons de l'Arxiu Municipal ha aparegut el text d'un Capbreu de la Cartoixa de Scala Dei, datat els dies 20, 21, 22, 23, 25, 26 i 27 de juliol de l'any 1491. Es tracta d'un quadern de mida foli, de nou fulls, guardat dins d'unes cobertes de paper, on hi ha escrit Capbreu de la Cartoxa i en lletra diferent, al marge esquerre «Cabreu de la Cartoixa». La conservació del paper i de la tinta és bona, i l'escriptura, encara que plena de correccions i esborranys, és regular en el seu traçat i fa ús de les abreviatures habituals de l'època. En la relació de prestacions, al marge esquerre de les divuit pàgines manuscrites, s'indica el tipus de pagament que es feia al monestir: el cens i el bovatge.

La lectura del text suggereix dades importants sobre els dominis senyorials de Scala Dei a Valls i sobre la situació de les partides del terme, així com de la configuració urbana de la vila. La novetat del document radica sobretot en la informació que aporta sobre la localització dels llocs que pagaven drets al monestir dins de la comarca vallenca $i$ de la vila.

Lexistència d'aquest domini senyorial és coneguda per la transcripció d'un privilegi de Ferran II, rei d'Aragó, inclòs en el document d'Establiment de la Lleuda de Valls, de l'any 1549, en el qual es descriu el procés d'adquisició d'alguns drets reials pel 
monestir de Scala Dei. Raimond Cescomes i Romeu Cescomes van lliurar al monestir l'any 1370 els drets reials que percebien a la ciutat i Camp de Tarragona, pel preu de 75.000 sous. Els Cescomes els havien obtingut per donació del seu parent, l'arquebisbe de Tarragona Arnau Cescomes, el qual els havia comprat a Bernat d'Olzinelles, tresorer del rei Pere IV, el 27 de desembre de 1342 .

... sane magestati nostre exposuit venerabilis et devotus religiosus, dilectus noster, prior monasterii Scale Dei, ordinis Cartusiensis, archiepiscopatus Tarracone, quod olim fuerunt per Raymundum Cescomes et Romeum Cescomes translati in dictum monasterium nonnulli redditus regales, qui percipiunt percipique solent in civitate Tarracone et in aliis locis campi Tarracone, precio videlicet septuaginta quinque mille solidorum, pro intrata cuiusdam stabilimenti facti et concessi per monasterium et conventum Scale Dei dicto Raymundo Cescomes de loco de Saydi.

Quos redditus prefati Cescomes adquisiverat a tunc archiepiscopi Tarracone, qui eo redditus, tamquam layca et privata persona, emerat a Bernardo de Olzinellis, legum doctori, thesaurario serenissimi regis Petri, divini recordii, precio videlicet quadraginta mille solidorum, prout de predicta vendicione constare vidimus per publicum instrumentum actum Tarracone, sexto kalendas januarii, anno Domini millesimo trecentesimo quadragesimo secundo...'.

La familia Cescomes va ser particularment important per a la vila de Valls. L'Arquebisbe Arnau Cescomes va pagar l'arranjament del mur i va concedir a la població la celebració d'una fira anual el mes de setembre?

Entre les clàusules de confirmació de l'esmentat privilegi de Ferran II s'especifica el nom de la localitat de Valls.

His igitur moti respectibus intuitu charitatis tenore presentis deque nostra certa sciencia deliberate et consulto dictos regios

' Josep Trenchs 1 OdenA, "La Lezda de Valls (Tarragona) 1549", Cuadernos de Historia Económica de Cataluña XIX, Barcelona, novembre 1978, pp. 155-189.

2 Josep Blanch, Arxiepiscopologi. 
redditus, qui percipiuntur percipi solent predictum monasterium in civitate Tarracone, villis de Vallibus, de Cambrils, de Reus, de Costanti, de Vilasicca de Solsina, de Montroig, de Riudoms, termino de Vilagrasa, campi de Tarracone et aliis locis eiusdem campi eidem monasterio laudamus, approbamus, ratifficamus et confirmamus... ${ }^{3}$.

Segons aquest privilegi i els que posteriorment van fer la reina D $^{\mathrm{a}}$ Joana, Carles I i Felip II, reis de Castella, en el mateix document d'Establiment de la Lleuda de Valls, Scala Dei tenia sobre Valls el dret alodial i emfitèutic, la vigilància en la recaptació de les imposicions, la capacitat de denunciar actes fraudulents i el control sobre qualsevol activitat comercial.

El Capbreu de Cartoixa de 1491 especifica les aportacions econòmiques dels veïns de Valls pels contractes emfitèutics que tenien concertats amb el monestir, entre els quals hi tenien, a més dels censos i bovatges, alguns lluïsmes i un homenatge. Es gravaven les possessions de terres en les partides de la comarca i també els albergs que habitaven els seus prestataris dintre de la vila. A les partides es pagaven censos $\mathrm{i}$ bovatges per tots els cultius de la terra vallenca, i a la vila, dintre del barri antic i al carrer de la Vilanova, en pagaven els edificis.

Lanàlisi detallada del cobrament dels drets monacals dóna idea de la riquesa del terme de Valls i del poder econòmic de Scala Dei en el segle xv. Les famílies antigues d'aquella vila rebien per herència i successió dels seus avantpassats unes propietats de les quals era senyor un monestir ben llunyà.

\section{DOMINIS DE SCALA DEI A LES PARTIDES}

Les partides subjectes al pagament de drets al monestir cobrien una àrea molt fèrtil entre el riu Francolí $\mathrm{i}$ la riera de Cabra. Des de la via general de Tarragona se situaven en un cercle envoltat per les poblacions de Vallmoll, Alió, el Pla de Santa Maria, Figuerola, Fontscaldes, Camí de Montblanc i Picamoixons.

Id. nota 1. 


\section{A Valls:}

La partida de l'Ermita.

La partida del Cami Nou, amb el Rec del Mas d'en Bas.

La partida d'Espinavesa, tocant al lloc de la Pobla, amb els Recs del Mas d'en Bas i Rec dels Cabanyals, vers Vallmoll i el riu Francoli.

La partida de la Freixa.

La partida de la Magdalena, tocant al Coll d'en Fula.

Vers Vallmoll:

La partida del Cabiscol, amb el Rec del Cabiscol.

La partida del Fontanals, amb el Rec dels Fontanals i el Rec del Cabiscol.

Vers Alió:

La partida de les Parellades del Rei, amb el Torrent del Catllar.

Vers el Pla de Santa Maria i Figuerola:

La partida de Palau de Reig, amb el Torrent de la Farrera.

Vers Fontscaldes:

La partida de la Plana d'en Berga.

La partida de la Verneda, amb el Torrent de la Farrera.

Vers Montblanc:

La partida de la Farrera, amb el Torrent de la Farrera, tocant a les partides de Palau de Reig i de la Verneda.

Vers Picamoixons:

La partida del Burgar, junt al Mas d'en Cabot.

La partida de Vergós, junt al Camí de l'Ermita (Sant Llorenç o Sant Jeroni).

En les prestacions de cada una d'aquestes partides, hi figura el nom del prestatari, la quantitat pagada, el tipus de cultiu i el nom del lloc, els límits amb altres partides o propietaris i el document mitjançant el qual pot disposar-se d'una terra a cens o a bovatge.

Aquestes noticies permeten fixar quins eren els indrets on estaven situats els masos, les deveses, els regadius, les vinyes, les oliveres, els erms i les garrigues. 
1 bovatge.

B. 9. Una vinya. 1 puieró. Document en forma pública.

\section{Partida del Cami Nou}

1 cens.

2. Una propietat. Laudemi.

\section{Partida d'Espinavesa}

1 cens i 2 bovatges.

Núm. 5: Una peça de terra. Laudemi.

Núm. 117: Un regadiu. Dos puierons menys un cóp d'ordi i un puieró de blat. Títol de successió.

145. Un regadiu, 3 puierons d'ordi i $1 / 2$ puieró de blat. Títol de donació.

Johannes Oliver confitetur et recognoscit se facere et prestare tres puyeronos ordei et medium puyeronum frumenti, in festo sanctorum Petri et Felicis, racione unius subrigui siti a Spinavesa. Affrontat vico Riparia de Francolino, carraria Molinera, vico de la Pobla. Titulo donacionis facte per Johannes Oliver, tempore sui matrimonii.

\section{Partida de la Freixa}

3 bovatges.

46. Una propietat. 1 puieró d’ordi. Títol de compra.

Item (Anthonius Montagut) et unum puyeronum ordei de bovatico, in festo santorum Petri et Felicis, racione unius propietate site al Frexa. Affrontat Bernardo Malafre, vico del Frexa et cum heredibus Anthonio Piquer a duabus partibus. Juravit se non habere instrumentum, ymo titulo empcionis facte ab 
Anthonio Piquer, socero suo et quod non habet instrumentum, ymo, etc.

57. Una peça de Terra. $1 \frac{1}{2}$ puierons d'ordi. Títol hereditari.

62. Una propietat de secà. $1 / 2$ quartera d'ordi. Títol de donació per núpcies.

La partida de la Magdalena

1 bovatge.

B. 10. Una masia. 6 puierons i 2 cóps d'ordi i 2 puierons de blat. Títol hereditari.

Guilermus Armengol, mercator ville de Vallibus, confitetur et recognoscit se tenere quandam masiam sitam in termino dicte ville, in parte vocata La Magdalena. Affrontat cum vico de Picamoxons et vico de la Armita, Francisco Loreda, Bartholomeo Carbonell, notario presentis scripti. Et tenetur ad prestacionem sex puyeronos et duos cops ordei de bovatico et duos puyeronos frumenti, ad mensura ville de Vallibus, in festo sanctorum Petri et Felicis. Juravit se non habere instrumenta aliqua, ymo titulo hereditario successisse Johannis Armengol, quondam, patris sui.

\section{La partida del Cabiscol}

2 bovatges.

B. 156. Un regadiu. 1 puieró d'ordi. Títol de compra.

B. 157. Un regadiu. 1 puieró d'ordi. Títol de successió.

Item (Berengarius Vicens) confitetur et recognoscit se facere unum puyeronum de bovatico ordei, racione unius subrigui siti al Cabiscol. Affrontat secum, Johanne Oliver, Anthonio Torres, Johanne Casquer et alveo del Cabiscol. Título successionis Berengarii Vicens successisse. 
11 bovatges.

B. 35. Un regadiu. 1 puieró d'ordi. Sense títol.

B. 36. Un regadiu. $1 \frac{1}{2}$ puierons d'ordi i $1 / 2$ puieró de blat.

B. 54. Un regadiu. 1 puieró d'ordi. Títol hereditari.

B. 55. Un regadiu. 4 puierons d'ordi. Títol hereditari.

B. 74. Una propietat de regadiu. $1 \frac{1 / 2}{2}$ puieró de blat. Títol de donació nupcial.

B. 75. Un regadiu. 1 puieró de blat. Títol de successió.

B. 76 . Un regadiu. $2 \frac{1}{2}$ puierons d'ordi $\mathrm{i} 1 / 2$ de blat. Sense títol.

B. 81 . Un regadiu. 8 puierons d'ordi. Títol de successió.

B. 131. Una propietat de regadiu. 1 puieró i 1 cóp de blat. Sense títol.

B. 134. Una propietat. 4 puierons d'ordi i 2 cóps de blat. Títol de donació nupcial.

Anthonius Cavos confitetur et recognoscit se facere et prestare anno quolibet in festo etc. quatuor puyeronos ordei et duos cops frumenti, racione unius propietatis site als Fontanals. Affrontat cum alveo dells Fontanals, Johanne Garcia, alveo del Vilar, secum. Titulo donacionis tempore nupciarum, facto Laurencie, uxoris sue, filie Andree Oliver.

B. 154. Un regadiu. 2 puierons d'ordi i 1 puieró de blat.

Partida de les Parellades del Rei

23 censos.

C. 16. Una vinya. 2 sous. Títol de donació.

C. 17. Una peça de terra. 18 diners. Títol de successió.

C. 18. Una peça de terra. 3 sous. Títol de donació.

C. 19. Una vinya. 2 sous. Títol segons capbreu.

C. 20. Una vinya. 3 sous i 6 diners. Títol «iurii vevitate consistere».

C. 21. Una vinya. 18 diners. Títol segons capbreu.

C. 51. Una propietat. 18 diners. Document no públic. 
C. 53. Una vinya. 7 sous i 6 diners. Per compra.

C. 78. Una peça de terra, 4 sous. Títol de donació nupcial.

C. 82 . Una propietat de secà. 3 sous. Títol de venda.

C. 83. Una vinya. 6 sous i 4 diners. Títol de compra.

C. 90 . Una propietat de vinya. 18 diners. Per compra.

C. 94 . Una vinya. 2 sous i 6 diners. Títol de donació per dot.

C. 98 . Una propietat de 2 quarterades. 5 sous i 6 diners. Títol de successió.

Bartholomeus Tosquela, agricultor, confitetur et recognoscit se facere et prestare anno quolibet, festo Sancte Crucis, cum omni dominio, Domino Regi, quinque solidos et sex denarios, racione unius propietate site a les Parelades del Rey, in quo sunt due quarterate. Affrontat cum Francisco Virgili, a duabus partibus, Jacobo Bernado et torrente del Catlar. Juravit titulo successionis Petri Tosquela, quondam, patris sui, ymo.

C. 110. Una propietat de vinya. 2 sous. Títol hereditari.

C. 111. Una vinya. 2 sous. Sense títol.

C. 122. Una vinya. 4 sous. Títol de donació nupcial.

C. 123. Una propietat de secà. 3 sous i 8 diners. Títol de donació nupcial.

C. 138. Una peça de terra. 4 sous. Títol de compra.

C. 140. Oliveres. 2 sous i 2 diners. Títol de compra.

C. 146. Una peça de terra. 5 sous, 8 diners i obol. Títol de successió.

C. 149. Una vinya. 3 sous. Títol en poder del notari.

C. 160. Una terra de secà. 5 sous i 8 diners. Títol de successió.

\section{Partida de Palau de Reig}

7 bovatges.

B. 66. Una masia. 3 quarteres, 5 puierons d'ordi i 1 quartera, 1 puieró i 1 cóp de blat. Títol de permuta.

B. 118. Una terra de secà. $1 \frac{1}{2}$ puierons d'ordi i 1 puieró de blat. Títol de successió. 
B. 119. Una propietat. $1 / 2$ cóp de blat $\mathrm{i} 1 / 2$ cóp d'ordi. Establiment.

B. 120 . Una propietat. $1 / 2$ cóp d'ordi i $1 / 2$ cóp de blat. Establiment.

B. 121. Oliveres. 2 puierons d'ordi. Títol en poder del notari.

B. 129. Una propietat. 5 puierons d'ordi. Títol de successió.

Petrus Barril, loci de Alione confitetur et recognoscit se facere et prestare quinque puyeronis ordei, racione unius propietate site a Palau de Reig, que possidetur una pars per Petrum Valla illud quod erat Johannis Sabaterii et id quod possidetur per Petrum Barril fuit Johannis Sabaterii. Affrontat cum vico de Figuerola, vico de Penafreyta, vico de Vilafranca et Mansi de Muletorum Petri Albinyana, qui olim fuit d'en Boras. Título successionis Petri Soler er Petrus Val titulo empcionis quam fecit a Johanne Sabaterii, Fiat ut supra.

B. 148. Una propietat. 2 puierons d'ordi. Títol de compra.

Partida de la Plana d'en Berga

2 bovatges.

B. 59. Una vinya. 3 puierons d'ordi. Títol de compra.

B. 155. Una vinya. $1 / 2$ puieró d'ordi. Títol de successió.

\section{Partida de la Verneda}

1 cens i 2 bovatges.

B. 89. Una peça de terra. 2 puierons d'ordi. Títol de compra.

B. 116. Un erm, abans vinya. $1 / 2$ puieró d'ordi. Títol de successió.

C. 127. Una propietat. 1 puieró d'ordi. Títol de successió.

Item (Jaume Anguera) nomine suo confitetur et recognoscit se facere et prestare anno quolibet unum puyeronum ordei de bovatico, racione unius propietate site a La Verneda. Affrontat cum Petro Cabrer, heredibus Johannis Radon, Torrente de la Farrera, vico de Fontibus Calidis seu de Miramar. Titulo predicto. 
3 bovatges.

B. 11. Una peça de terra. $1 / 4$ de puieró d'ordi i $1 / 4$ de puieró de blat. Títol hereditari.

B. 45. Oliveres. 1 puieró d'ordi. Títol de donació.

B. 135. Una propietat de secà. 1/2 cóp d'ordi. Títol de compra.

Bernardus Teixidor confitetur et recognoscit se facere et prestare anno quolibet in festo sanctorum Petri et Felicis, serenissimo domino Regi, medium cop ordei, racione propietatis unius sicani site a La Farrera. Affrontat cum Anthonio Bartholomei, Petro Furtuny, Ludovico Martorel, Johanne Ginera, vico Montis Albi. Pertinet sibi titulo empcionis quam fecit a Petro Albinyana, Mansi Muletorum ut constat in instrumento publico acto in dicta villa de Vallibus, prima die marcii, anno a Nativitate Domini M CCC LXXX, et clauso per discretum Johannem Mataler, quod notarius est, et fit mensio de dicto bovatico in instrumentis.

Partida del Burgar

C. 86. Una devesa. 1 lliura de cera. Títol segons capbreu.

Item (Berengarius Balcells) confitetur et recognoscit se facere et prestare anno quolibet in festo Nativitatis Domini, dominacioni communi, omni dominio, unam libram cere bone, racione uinius devesie site in termino dicte ville, in parte vocata Lo Burgar. Affrontat cum heredibus Guilermi Armengol, Johanne Oller et duabus partibus cum dicto Berengario Balcells. Et pertinet sibi... est similis in capibrevio, in titulo 23 .

\section{La partida del Vergós}

3 bovatges.

B. 58. Una vinya. $1 \frac{1}{2}$ cóp de blat $\mathrm{i} 1 / 2$ d'ordi. Títol de permuta.

B. 162. Una propietat. 2 cóps d'ordi i 1 cóp de blat. Títol de donació nupcial. 
B. 166. Una possessió, part de terra campa i part de vinya. 1 puieró d'ordi. Títol en poder del notari.

Petrus Oller, agricultor, confitetur et recognoscit se facere unum puyaronum ordei de bovatico, racione unius possessionis partim terre campe et partim vinee plantati, siti a Vergos. Affrontat cum Johanne Rabuster, Andrea Talavera, Johanne Baffarull, vico de Picamoxons. Juravit quod emit a Johanne Baffarull et quod instrumentum est in notario.

En el Capbreu també s'esmenten propietats i terres de les viles circumdants, en les quals tenien drets els monjos de Scala Dei.

\section{Bassa de la Bona}

1 bovatge.

B. 31. Una vinya. 1 puieró d'ordi. Títol de compra.

Petrus Gili, parator pannorum, confitetur et recognoscit se facere et prestare anno quolibet, in festo sanctorum Petri et Felicis, dominacioni communi, unum puyeronum ordei bovatico, racione unius vinee site a la Bassa de la Bona, al Camp de la Vilanova. Affrontat cum discreto Berengario Martini, Marcho Gavaldana a duabus partibus et via publica del Plano. Juravit titulo empcionis successisse et non est sibi instrumentum.

\section{Camp d'en Gausent}

\section{1 bovatge.}

B. 26. Un regadiu. 1 puieró d'ordi. Títol de successió.

Petrus Ferarrii, mercator confitetur et recognoscit se facere et prestare unum puyeronum ordei de bovatico, in festo sanctorum Petri et Felicis, dominacioni communi, racione cuiusdam terre troci de solrech, siti al Camp d'en Gausent. Affrontat cum Jacobo Anguera, secum et cum quodam alio trocio terre ibidem contiguo, quod tenetur per beneficium Sancte Margarite. Dixit sibi 
pertinere titulo successionis Petri Ferrarii, quondam, patris sui et dicto suo patri, prout in capibrevio.

Camp de la Vilanova

1 bovatge.

B. 31. Una vinya. 1 puieró d'ordi. Títol de compra.

Cap de la Vilanova

1 bovatge.

B. 27. Una terra de secà. $1 \frac{1 / 2}{2}$ puieró de blat i 2 puierons d'ordi. Títol segons capbreu.

Item (Petrus Ferrarii) et quandam preciam terre sicani sitam al Cap de Vilanova. Affrontat cum Petro Ferrarii in tribus partibus, secum; et facere unum puyeronum et medium frumenti et duos ordei de bovatico, anno quolibet, in festo sanctorum Petri et Felicis. Juravit prout supra et in capibrevio.

Coll d'en Fula

2 bovatges.

B. 8. Una vinya. 1 puieró d'ordi. Títol de compra.

Jacobus Crivileres, agricultor, confitetur et recognoscit se tenere serenissimo domino Rege ad prestacionem unius puyeroni ordei de bovatico, in festo sanctorum Petri et Felicis, quandam vinem sitam al Col d'en Fula. Affrontat cum Jacobo Ferrarii, Johannis Caus, Bartholomeo Torres et via publica. Juravit se titulo empcionis successisse, quam fecit a Jacobo Sarra, sutore, et quod instrumentum non fuit sibi factum.

B. 152 . Una propietat. $2 \frac{1}{2}$ puierons d'ordi. Titol hereditari. 


\section{Los Comalors}

1 cens.

C. 103. Una propietat a Picamoixons. 11/2 puieró de blat. Títol de permuta.

Anthonius Catala, loci de Picamoxons confitetur et recognoscit se facere et prestare anno quolibet, in festo sanctorum Petri et Felicis, domino Regi, unum puyeronum et medium frumenti de bovatico, racione unius propietatis site in dicto loco de Picamoxons, in loco apellato Los Comalors. Affrontat cum Berengario Balcells et a tribus partibus cum quibusdam garrigiis. Juravit titulo permutacionis facte inter Petrum Catala, fratrem suum.

\section{Coll dels Sants}

2 bovatges.

B. 43 . Una terra campa, abans vinya. $2 \frac{1}{2}$ puierons d'ordi. Títol de compra.

Petrus Oliver, agricultor ville de Vallibus, confitetur et recognoscit se facere et prestare anno quolibet, in festo sanctorum Petri et Felicis, serenissimo domino Regi, duos pueronos et medium ordei ad mesuram Ville de Vallibus, racione cuiusdam terre campe olim vinee plantate al Col dels Sants. Affrontat secum, Anthonio Segura, Petro Puyalt, heredibus d'en Torroella. Juravit se titulo empcionis successisse et admissit instrumentum ymo etc.

B. 44. Una peça de terra. 1 cóp d'ordi i 1 cóp de blat. Títol de successió.

\section{Mas d'en Bosc}

1 bovatge.

B. 128. Una peça de terra. 5 puierons d'ordi. Títol de compra.

Laurencius Monlaho, senior, confitetur et recognoscit se facere et prestare duos puyaronos et medium ordei de bovatico, una 
cum alia pecia terre contigua, que nune possidetur per Johannem Plana, de Alione, duos et medium ordei et sunt in summam quinque puyaronos et una per alia obligata et propietas dicti Laurencii Monlaho. Affrontat cum Marcho Vives, censualibus Raymundi Armengol, vico de Vilafranca, alveo d'en Boras, quod venit del Mas d'en Bosch, Laurencii Monlaho...

\section{Mas d'en Cabot}

3 bovatges.

B. 115. Una propietat. 1 puieró d’ordi. Establiment.

Dalmacius Duffort, parator dicte ville, confitetur et recognoscit se facere et prestare anno quolibet, in festo sanctorum Petri et Felicis, serenissimo domino Regi, unum puyeronum ordei, racione unius proprietatis site al Mas d'en Cabot. Affrontat cum vinea Sancii Anthonii, cum alveo quod ibat ad mansum d'en Bertran, que olim erat Johannis Berengarii, torrente mansi d'en Cabot, Petro Bosch et Argamassa de la Risclosa. Juravit titulo stabilimenti...

B. 137. Una vinya. $1 \frac{1 / 2}{2}$ puieró d'ordi. Per compra.

B. 163. Una peça de terra, abans vinya. 1 puieró.

\section{Molf $i$ Rec d'en Caus}

1 laudemi.

L. 126. Una peça de terra. Laudemi. Títol de successió.

Jacobus Anguera, curator testamentarius, ut asserit Marchi Anguera, fratris et germani sui confitetur et recognoscit dictum pupillum habere, tenere quandam peciam terre, sitam supra Molendinum d'en Caus. Affrontat cum Anthonio Anguera, Anthonio Belvell, alveo Molendini d'en Caus, que tenetur ad prestacionem laudimiii, serenissimo domino Regi. Juravit titulo successionis Jacobi Anguera, quoandam, patris dicti pupilli successisse. 
1 bovatge.

B. 40. Una vinya. 1 puieró. Sense títol.

Petrus Puig, agricultor, confitetur et recognoscit se facere anno quolibet, in festo sanctorum Petri et Felicis, unum puyeronum ordei, serenissimo domino Regi, racione cuiusdam vinee et eremi site in parte vocata lo Mas d'en Celada. Affrontat cum Anthinio Armengol, Petro Jover, Raymundo Armengol, Marcho Mateu et via publica. Juravit se non habere instrumenta aliqua, ymo.

\section{Fontscaldes}

2 censos i 3 bovatges.

C. 113. 1 lliura de cera. Títol de successió.

C. 114. Una propietat. 6 diners. Establiment.

C. 141. Una propietat part vinya i part terra campa. 1 puieró i 1 cóp d'ordi. Títol de successió.

Gabriel Gaya, loci de Fontis Calidis confitetur et recognoscit se facere et prestare anno quolibet, in festo sanctorum Petri et Felicis unum puyeronum et unum cop ordei, racione hunius pecie terre partim vinea plantati et partim campi siti prope dictum locum de Fontis Calidis. Affrontat cum Laurencio Mestre dicti loci et vico de Vilafranca, virtute successionis Johannis Gaya, quondam, patris sui.

B. 142. Una terra de secà. 1 cóp d'ordi. Títol de successió.

B. 159. Una terra de secà. 1 puieró d'ordi. Títol de donació.

\section{Mas de Mulets}

2 bovatges.

B. 79. Una propietat. 5 puierons d'ordi i $1 / 2$ puieró de blat. Títol de successió. 
B. 133. Una propietat. 5 puierons d'ordi i $1 / 2$ puieró de blat. Títol de successió.

Anthonius Grimau, Mansi Muletorum, confitetur et recognoscit se facere et prestare quinque puyeronos ordei et medium puyeronum frumenti, racione unius proprietatis site prope dictum Mansi, de qua medietas possidetur per Petrum Albinyana et tenetur ad prestacionem medietatis predicti bovatici. Affrontat cum dicto Albinyana a duabus partibus, Anthonio Grimau minore et via publica Montis Albi. Propietas dicti Albinyana cum Anthonio Grimau seniore, Anthonio Grimau juniore, secum et dicto vico. Titulo successionis Francisci Grimau et Bernardi Albinyana, patrum predictorum. Fiat ut in capibrevio in titulo 9.

\section{La Mola}

1 bovatge.

B. 48. Una masia. $1 / 2$ quartera de blat. Títol de compra.

Anthonius Bertran, alias Romeu, agricultor, ville de Vallibus, confitetur et recognoscit se facere et prestare mediam quarteram de frumenti ad mensuram ville de Vallibus, in festo sanctorum Petri et Felicis, racione cuiusdam masie, cum suis terris, site in loco de La Mola, qui olim fuit d'En Johan Berenguer. Affrontat cum Torrente de La Mola, vico de Montblanc, Anthonio Johanni Alamany, discreto Johanne Andrea, presbiteri. Juravit titulo empcionis successisse, cum inde fecit a curatoribus bonorum dicti Johannis Berengarii et quod est in tabellionatu, ymo.

\section{Picamoixons}

11 bovatges.

B. 85 . Una heretat. 3 puierons d'ordi i $1 \frac{1}{2}$ puierons de blat. Títol de successió.

B. 92. Un corral. 1/2 puieró d'ordi. Titol de successió.

B. 93. Un hort. 11/2 cóp d'ordi.

B. 103. Una propietat. $1 \frac{1}{2}$ puieró de blat. Títol de permuta.

B. 104. Una peça de terra. $1 / 2$ puieró d'ordi. Títol de permuta. 
Item (Anthonius Catala) confitetur et recognoscit se facere et prestare anno quolibet, in dicto festo, de bovatico medium puyaronum ordei, racione unius pecie terre site in dicto loco (Picamoxons), in parte vocata La Riberaça. Affrontat cum rivo de Francholini, Anthonio Cistarer, Gabriele Cistarer et vico generali, quod itur ad villam Montis Albi. Titulo predicto.

B. 105. Un hort. 3 puierons d'ordi. Títol de permuta.

B. 106. Un hort. 11/2 cóp d'ordi. Títol hereditari.

Anthonius Cisterer, loci de Picamoxons, confitetur et recognoscit se facere et prestare anno quolibet, in festo sanctorum Petri et Felicis, unum cop et medium ordei quendam ortum situm in dicto loco de Picamoxons. Affrontat a duabus partibus cum Berengario Balcells. Torrente del Canar, secum. Juravit titulo hereditario Francisci Cistarer, patris sui successisse et quod non habet instrumentum.

B. 107. Un hort. 2 puierons d'ordi i $1 / 2$ puieró de blat. Títol hereditari.

Item (Anthonius Cistarer) et quendam ortum situm in dicto loco, in parte vocata loco del Roures. Affrontat cum Berengario Balcells, vico de Alila, et secum a duabus partibus. Tenetur ad prestacionem duorum puyaronum ordei et medium puyaronum frumenti. Titulo predicto.

B. 108. 1 puieró d'ordi. Títol hereditari.

B. 143. Una propietat. $1 / 2$ puieró d'ordi. Títol de compra.

B. 144. Una garriga. $1 / 2$ cóp d'ordi.

Item (Berengarius Balcells) et medium cop ordei, racione unius garigua. Affrontat Anthonius Catala, vico de Fonscaldes et Francisco Cistarer, Torrente de $\mathrm{Na}$ Balaguera.

\section{La Pobla}

1 bovatge.

B. 164. Una peça de terra. 1 puieró i 1 1/2 cóp d’ordi. Sense títol. 
Maria Cerdana, vidua, uxor Petri Cerda, quondam, confitetur et recognoscit facere in festo sanctorum Petri et Felicis, serenissimo domino Regi, de bovatico unum puyeronum et medium cop ordei, racione unius terre trocii subrigui, siti a La Pobla. Affrontat cum camino Molineris de la Granja de Dolt de Lops, cum Petro Giner et cum Possessioni qui antiquitus fuit Johannis Oller, Bernardo Domenech. Juravit non habere.

\section{Pont de Goi}

1 bovatge.

B. 151. Mitja Masia. 1 "puya» i 3 cóps. Títol hereditari.

Petrus Cistarer, agricultor, confitetur et recognoscit se facere et prestare anno quolibet, in festo sanctorum Petri et Felicis, serenissimo domino Regi, unum puya et tres cops ordei, racionem medietatis masie site a Pont de Goy. Affrontat cum via generali de Tarragona et a tribus partibus cum heremis. Juravit titulo hereditario, quondam, Gabrielis Cistarer, quondam, patris sui, successisse.

\section{Pont de na Pellicera}

1 bovatge.

B. 61. Una propietat, mitja quarterada és de rec i la resta, de secà. Títol de successió.

Guilermus Anguera, sartor dicte ville confitetur et recognoscit se facere et prestare anno quolibet, in festo sanctorum Petri et Felicis unum puyeronum de forment de bovatico, serenissimo domino Regi, racione unius proprietatis site al Pont de Na Pelicera, est media quarterata de regui et residua parte de sicano. Affrontat cum Johanne Alamany et a tribus partibus via publica. Juravit titulo successionis patris sui successisse et si invenerit aliqua instrumenta etc. 
1 bovatge.

B. 158. Un regadiu. 1 puieró de blat. Títol de successió.

Item (Berengarius Vicens) confitetur et recognoscit anno quolibet, unum puyaronum frumenti, racione unius subregui siti als Cabayalls. Affrontat cum Gabriele Olesa, Johanne Torradamer, Vico Honorum et a duabus partibus cum alveo dells Cabanyalls. Pertinet titulo predicto.

\section{Tarragona}

1 cens.

C. 7. Un moli. 3 quarteres. Per compra.

Item (Anthonius Johannes Nadal) et quoddam molendinum situm in termino dicte ville, satis prope vicum de Tarragona, cum nonnullis terris, honoribus et possessionibus. Affrontat cum Johanne Safortesa, cum Petro Jover et via publica et tenet ad prestacionem trium quarteriarum ordei, unam domino Archiepiscopo, alia preposito mensis septembris et alteram Monasterio Scale Dei, alias Cartoixa. Juravit quod emit a Marcho Pasquali. Est in notario instrumentum.

En aquesta enumeració de partides i propietats solament s'esmenten aquelles sobre les quals el monestir de Scala Dei percebia drets, encara que en el Capbreu se'n citen altres que són els límits de les possessions gravades pels censos o bovatges. D'aquestes últimes, se'n farà relació a l'índex.

\section{DOMINIS DE SCALA DEI DINS LA VILA DE VALLS}

Els monjos del monestir de Scala Dei posseïen albergs en tots el nuclis urbans de Valls, en el barri més antic, a la Vilafranca, a la Pobla de Santa Anna, però sobretot a la Vilanova, un carrer ja conegut el segle xiII i que es va anar poblant durant el segle 
XIV, en un moment demogrảfic important per al desenvolupament de la vila. Aquest carrer s'eixamplava sobre una partida de vinyes i terres, anomenada Cap de la Vilanova ${ }^{4}$ o també Camp de la Vilanova. Corria a la vora de l'Andador del Mur, que era el corredor existent entre el Mur i la fila de cases de la Vilanova ${ }^{5}$. Al segle xv els conflictes socials van frenar el creixement demogràfic $\mathrm{i}$ urbanístic fins a tal punt que van arribar a tenir conseqüències greus en les relacions entre la Cartoixa i el municipi de Valls. L'any 1484 es va promoure un litigi davant del Procurador Reial a Tarragona, per causa dels interessos oposats dels litigants en el cobrament de la lleuda per l'entrada de mercaderies. Aquest tribut havia estat imposat per l'arquebisbe Pere d'Urrea i el prior de Scala Dei, Antoni de Torres ${ }^{6}$. L'any 1491, data del Capbreu, durant l'arquebisbat de Gonzalo Fernández de Heredia, les tensions ja havien minvat.

Làrea d'imposicions de tributs pel monestir es concentrava en els nuclis del barri antic i del carrer de la Vilanova. Al barri antic: els carrers de la Carnisseria o del Portal Nou, pròxima al barri dels Jueus; de la Sabateria i el carrer d'en Simó. Limitaven amb la Vilanova les zones del Pati del Castell, la plaça del Castell o de la Vilanova i la plaça de la Peixateria; solament pagaven drets la Vilanova i la plaça del Castell.

Descripció d'habitatges per carrers:

Barri Antic

Carrer de la Carnisseria o del Portal Nou

4 censos.

C. 4. Una casa. 11 sous i 4 diners.

C. 41. Una casa. 9 sous. Títol de successió.

C. 42 . Una casa. 5 sous. Títol en poder del notari.

4 Gabriel Secall i Goell, «Notes locals de Topografia Urbana medieval», Cultura, Valls, desembre 1978, pp. 87-90.

${ }^{5}$ Gabriel Secall 1 Goelı, "Del Pati del Castell a la Plaça de la Palma», Cultura, Valls, desembre 1980, pp. 26-31.

- Josep Trenchs I Odens, "La Lezda de Valls (Tarragona) 1549", Cuadernos de Historia Económica XIX, Barcelona, novembre 1978, pp. 158-189. 
Petrus Raymundi, faber dicte ville, confitetur et recognoscit se facere $V^{e}$ solidos in festo Nativitatis Domini, cum omni dominio, dominacioni communi, racione domus site in vico de la Carniceria aliter del Portal Nou. Affrontat cum Petro Cabrer, Anthonio Ferrarii, vico dells Jueus, Barthomeva Fustera et via publica. Juravit se non habere instrumenta cum fuit in scribania, ymo.

C. 130. Una casa. 18 diners. Establiment.

\section{Carrer de la Sabateria}

6 censos.

C. 1. Una casa. 16 sous. Títol de successió.

C. 6 . Una casa. 8 sous.

C. 100 . Una botiga. 6 sous i 8 diners.

Bartholomeus Radon, habitator ville de Vallibus, confitetur et recognoscit se facere et prestare in festo Nativitatis Domini, cum omni dominio, sex solidos et octo denarios, racione unius botigie site in dicta villa, in vico de la Sabateria. Affrontat Petro Monlaho, secum a duabus partibus, et via publica. Juravit titulo hereditario Bartholomei Radon, quondam, avi sui, et dicto Bartholomeo Radon titulo empcionis, quod fecit ab Anthonio Piquer. Ostendit quoddam publicum instrumentum, actum in dicta villa, $\mathrm{V}^{\mathrm{a}}$ die februarii, anno a Nativitate Domini M CCC L VIII', sed non clauso nech dominii racione, firmato.

C. 101. Una casa. 4 sous i 8 diners. Títol hereditari.

C. 125 . Una casa. 6 sous. Per compra.

C. 147 . Una casa. 8 sous i 4 diners. Per compra.

\section{Carrer d'en Simó}

4 censos.

C. 34 . Una casa. 12 diners. Document públic, fet el 13 de març de 1489. 
C. 87. Una casa. 5 sous. Títol de successió.

C. 95 . Un celler, 8 diners. Títol hereditari.

Laurencius Tamarit confitetur et recognoscit se facere et prestare anno quolibet, in festo Nativitatis Domini, cum omni dominio, dominacioni communi, octo denarios de censu, racione uinus cellarii siti in vico d'En Simó. Affrontat cum Latzaro Carbonell, cellario prepositi, secum ét via publica. Juravit titulo hereditario Laurencii Tamarit quondam, patris sui, successisse.

C. 96. Una casa, 4 diners. Títol hereditari.

\section{La Vilanova}

Plaça del Castell

1 cens.

C. 33. Un corral. 15 diners. Títol de venda.

Item (Anthonius Armengol) et quindecim denarios, dominacioni communi, racione de hun corral siti prope platea del Castel, aliter de Vilanova. Affrontat cum Bernardo Jofre, Laurencio Montagut, Andatore Muri, via publica. Ostendit quoddam publicum instrumentum vendicionis sibi factum per Anthonium Giner, textorem lini, sed non clausum nech dominii racione firmatum, actum in villa de Vallibus a XVIIII de maig, anni M CCCC LXIIII.

\section{Carrer de la Vilanova}

40 censos.

C. 3 . Una casa. 22 diners. Títol hereditari.

C. 12 . Una casa. 2 sous i 4 diners. Document públic fet l'any 1424.

C. 14. Una casa. 22 diners i òbol.

C. 15 . Unes cases. 15 diners. Document públic fet l'any 1482.

C. 22 . Una casa. 2 sous i 11 diners. Títol segons capbreu.

C. 23 . Una casa. 7 sous i 10 diners.

C. 29. Una casa. 2 sous i 3 diners. Títol de venda. 
Petrus Marchii, parator ville de Vallibus confitetur et recognoscit se facere et prestare anno quolibet, in festo Nativitatis Domini, dominacioni communi, cum firma, francha etc. duos solidos et tres denarios, racione domus site in vico de Vilanova. Affrontat cum Raymundo Armengol, domus carcellarie, Andatore Muri et via publica. Ostendit quoddam publicum vendicionis instrumentum inde sibi factum per venerabilem Bernardum Calba, Ordinis Predicatorum, actum in villa de Vallibus, $\mathrm{III}^{\circ}$ die februarii, anno a Nativitate Domini M CCCC LXXX et clauso per discretum Johannem Mataler, notario dicte ville, et dominii racione firmatum per discretum Anthonium Mulner, procuratorem reverendissimi domini Archiepiscopi.

C. 30. Una casa. 15 diners. Títol segons capbreu.

C. 33. Un corral. 15 diners. Títol de venda.

C. 47 . Una casa. 12 diners. Títol de donació nupcial.

C. 49. Una casa. 15 diners. Títol hereditari.

C. 50. Una casa. 7 diners i obol. Títol hereditari.

C. 52 . Una casa. 13 diners. Títol de permuta.

C. 56 . Una casa. 2 sous i 3 diners. Títol de donació nupcial.

C. 60 . Una casa. 15 diners. Títol de compra.

C. 63. Una casa. 22 diners. Títol de venda.

Petrus Montagut, agricultor, confitetur et recognoscit se facere et prestare anno quolibet, in festo Nativitatis Domini, dominacioni communi, cum omni dominio, viginti duos denarios, racione domus site in vico de la Vilanova. Affrontat secum, vico del Abaurador, Petro Jover, Petro Castri. Ostendit quoddam publicum vendicionis instrumentum inde Johanni Montagut per Anthonium Belver factum, sed non clausum nech dominii racione firmatum, sed in sui publica forma redactum, actum in villa de Vallibus, XXVIIII ${ }^{\mathrm{a}}$ mensis aprilis, anno a Nativitate Domini M CCC LXXX V०.

C. 64. Una casa. 15 diners. Títol de venda.

C. 65 . Una casa, 6 diners. Títol de compra.

C. 67. Una casa. 2 sous i 6 diners. Títol de successió.

C. 68. Una casa. 15 diners. Títol de successió.

C. 69. Una casa i una cuina, tocant a la casa principal. 2 diners. Títol de successió. 

C. 70. Un corral. 10 diners. Títol de successió.
C. 71 . Una casa principal. 18 diners. Títol de successió.
C. 72. Una casa. 18 diners i òbol. Títol de transacció.
C. 73. Un corral. 3 diners i òbol. Títol de transacció.
C. 77 . Una casa. 6 sous. Títol de compra.
C. 84 . Una casa. 10 diners i laudemi.
C. 88 . Una casa dividida per transacció. 16 diners. Títol de compra.
C. 91. Una casa. 10 diners. Títol de successió.
C. 97 . Una casa. 6 diners i òbol. Títol de donació nupcial.
C. 99 . Una casa. 7 diners i òbol. Sense títol.
C. 102. Una casa. 10 diners. Títol de compra.
C. 109. Una casa. 10 diners. Títol de Pere Jofre.

Bernardus Jofre confitetur et recognoscit se facere et prestare anno quolibet, in festo Nativitatis Domini, cum omni dominio, decem denarios, racione domus site in vico de Vilanova. Affrontat cum via publica Bartholomeo Torres, Johanni Blasco, Eclesia de Santa Ana. Titulo a Petro Jofre pertinere.

C. 112. Una casa. 20 diners. Per compra.

C. 124. Una casa. 22 diners. Títol de donació nupcial.

C. 132. Una casa. 2 sous i 6 diners. Títol hereditari.

C. 136. Una casa. 15 diners. Títol de compra.

C. 139. Una casa. 7 diners i obol.

C. 150. Una casa. 15 diners. Títol de compra.

C. 165. Un corral d'ovelles. 10 diners. Per compra.

En la fixació de límits d'aquests albergs s'esmenten indrets molt interessants de la vila antiga de Valls: l'Andador del Mur, l'Abeurador, el Cementiri, prop del carrer d'en Simó, la Presó, el carrer del Pes de la Farina, el Pati del Castell, la plaça de la Peixateria i l'església de Santa Anna, a la Pobla. Però Scala Dei no exercia domini sobre cap d'ells.

La Vilafranca era abans del segle xIV una explanada travessada pel Torrent de la Vilafranca, que es va transformar en via pública, sobre la qual Scala Dei hi tenia un sol prestatari?

'Gabriel Stcall. I Gueut, «Aportació Històrica sobre les vies públiques dels 


\section{LLIURAMENT DELS CENSOS I BOVATGES}

Els censos de les partides o propietats de Valls pagaven als monjos de la Cartoixa amb la moneda corrent de l'època, sempre amb sous, diners i obols, per la festa de Nadal. Les quantitats lliurades oscil.laven entre un minim de 3 diners per un corra] al carrer de la Vilanova, i un màxim de 16 sous per una casa al carrer de la Sabateria. Per fer una comparança del valor real d'aquests pagaments, al segle xv el preu d'un jornal de mula era de 2 sous diaris.

Els bovatges es pagaven en quarteres, puierons i cóps d'ordi, blat o de tots dos grans. El lliurament mínim era de mig cóp per una terra de secà a la Partida de la Farrera; el màxim, de 3 quarteres d'ordi per un molí fora muralles, vora de Tarragona. Aquestes quantitats es lliuraven en les festes de Sant Pere i Sant Fèlix, segons la mesura de Valls, que representava quasi un quart de puieró més per quartera, comparada amb la de Tarragona.

Una altra forma de pagament era a pes de cera. Per una devesa a la Partida del Burgar es va pagar una lliura de cera.

També es pagaven llü̈smes per terres o cases alienades. En aquest any de 1491 estaven grabats a lluïsme un regadiu al Clot d'en Segura, per la qual cosa es va presentar un títol de venda redactat a Reus; una terra campa a les Parellades del Rei i una casa a la Vilanova, per la qual es va mostrar un títol de permuta.

En tot el text només hi ha un sol acte d'homenatge:

H. 13. Johannes Saforteça prestitit sacramentum et homagium ore et manibus multum magnifico Uguoni de Mediavilla etc., in presencia mei Bartholomei Carbonelli et Francisci Sarra, Petri Jope (sic).

voltants de la Carnisseria, de la Plaça de l'Oli i del Portal d'en Llobet», Cultura, Valls, octubre 1981, pp. 17-25. 


\section{ELS TÍTOLS DE POSSESSIÓ}

En la fórmula final de cada prestació s'escriu el títol de possessió, que solia ser per successió i herència, donació per Capítols Matrimonials, venda, compra, permuta i per establiment.

Alguna és curiosa i, d'altra banda, freqüent en el text:

B. 9. Ostendit quoddam publicum instrumentum non est in sua publica forma redactum.

B. 35. Juravit se non habere instrumentum.

B. 119. Juravit quo fuit sibi stabilitum per.

C. 122. Titulo donacionis facte per Petrum Bosch eius uxoris tempore suarum nupciarum.

Es podrien treure altres conclusions del text del Capbreu de la Cartoixa de l'any 1491, sobretot pel que fa a l'onomàstica i a la lingüística. També es podria fer un estudi de la genealogia a partir de la relació amb els avantpassats que apareix en la presentació dels títols dels usufructuaris d'una propietat o alberg.

\section{INDEX DE PARTIDES, PROPIETATS I LÍMITS}

L'ABEURADOR. (Límit de la Vilanova), C.63. ALILA, Lloc d'. (Limit de Picamoixons). V. ILLA, L'.

ALIÓ. (Límit de les Parellades del Rei, de la Vilafranca i del Rec d'en Borràs). C.17, C.18, C.37, C.78, C.110, B.128, C.146, C.149.

ANDADOR DEL MUR, (Límit de la Vilanova i plaça del Castell). C.3, C.12, C.14, C.29, C.33, C.49, C.52, C.55, C.60, C.65, C. 91, C.102, C.124, C. 165 .

ARGAMASSA DE LA RESCLOSA. (Límit del Mas d'en Cabot). C.115.

BACÍ DELS POBRES VERGONYANTS. (Límit de la Vilanova). C.112.

BALAGUERA, Torrent de na. (Límit de Fontscaldes). B.144. BAS, Rec d'en. (Límit de l'Espinavesa i del Cami Nou). C.2, C.25. BERGA, Plana d'en. (Partida). B.59, B.155. 
BERTRAN, Masia d'en. (Límit del Mas d'en Cabot). C.115. BONA, Bassa de la. (Propietat). C.31.

BORRẢS, Rec d'en. (Procedeix del Mas d'en Bosc). B.128.

BOSC, Mas d'en. (Lloc de procedència del Rec d'en Borràs). B.128.

BURGAR, El. (Partida i límit de Mas de Mulets i de Mas d'en Cabot). B.79, B.86, B.137.

CABANYALS, Rec dels. (Propietat i límit de l'Espinavesa). B.117, B.158.

CABISCOL, Partida i Rec del límit dels Fontanals). B.54, B.156, B.157.

CABOT, Mas d'en. (Propietat i límit amb la Plana del Burgar). C.115, B.137, B.163.

CAMÍ DE LERMITA. (Partida). B.9.

CAMÍ GENERAL A PICAMOIXONS. (Límit d'una propietat a la Riberassa de Picamoixons). B.104.

CAMÍ NOU. (Partida). C.2.

CANAR, Camí del. (Límit i Picamoixons). B.106.

CARME, Rec del. (Límit de la Partida del Castell). B.161.

CARNISSERIA, Carrer de la. C.4, C.41, C.42, C.130.

CASTELL, del. (Partida). B.80, B.161.

CASTELL, Pati del. (Límit a la Vilanova). C.64, C.91.

CASTELL, Plaça del. C.33.

CASTELL, Rec del. (Límit de la Partida del Castell). B.161.

CATLLAR, Torrent del. (Límit de les Parellades del Rei). C.20, C.98, C.111, C.137, C.146.

CAUS, Rec i Molí d'en. (Partida). C.38, L.126.

CELADA, Mas d'en. (Propietat). B.40.

CEMENTIRI. (Límit del carrer d'en Simó). C.96.

CLOT d'en SEGURA. (Propietat). C.38.

COMALORS, Los. (Propietat a Picamoixons). C.103.

ERMITA, L'. (Limit de la Magdalena i del Coll d'en Fula). B.9, B.10, B.152.

ESPINAVESA, L. (Partida). C.5, B.74, B.117, B.145.

FARRERA, La. (Partida i limit de la Verneda). B.11, B.45, B.127, B.135.

FARRERA, Torrent de la. (Límit a Palau de Reig). B.45, B.66. 
FIGUEROLA. (Límit de Palau de Reig). B.129, B.148.

FONTANALS, Els. (Partida). B.35, B.36, B.54, B.55, B.74, B.75, B.76, B.81, B.131, B.134, B.154.

FONTANALS, Recs dels. (Límit de l'Ermita). B.35, B.36, B.74, B.75, B.81, B.134, B.153., B.154.

FONTSCALDES. (Propietats i límit de la Verneda). C.113, C.114, C.115, B.127, B.141, B.142, B.159.

FORN DE COURE PA. (Propietat). C.114.

FRANCOLI, Riu. (Limit de la Riberassa de Picamoixons i de l'Espainavesa). C.104, B.145.

FREIXA, El. (Partida), B.46, B.57, B.62.

FULA, Coll d'en. (Propietat). B.8, B.152.

GAUSENT, Camp d'en. (Propietat). B.26.

GRANJA DE DOLDELLOPS. (Límit del Freixa i del Burgar). B.164.

ILLA, L'. (Límit de Picamoixons i del lloc dels Roures). B.92, B.107.

JUEUS, Barri dels. (Límit de la Carnisseria), C.41, C.42.

LILA, V. ILLA, L.

MAGDALENA, La. (Partida). B.10.

MAS DE MULETS (Propietat i límit de la via de Montblanc i de Fontscaldes). B.79, B.133, B.159.

MIRAMAR, Lloc de. (Límit de la Verneda). B.89, B.116.

MOLA, La. (Propietat). B.28, B.48.

MOLA, Torrent de la. (Límit de la Mola). B.28.

MONTBLANC. (Límit de Mas de Mulets, de Picamoixons i de la Farrera). B.79, B.105, B.133, B.135.

PALAU DE REIG. (Partida). B.66, B.118, B.119, B.120, B.121, B.129, B.148.

PARELLADES DEL REI, Les. (Partida). C.16, C.17, C.18, C. 19 , C. 20 , C. 21 , C. 51 , C. 53 , C. 78 , C.82, C.83, C. 90 , C.94, C. 98 , C.110, C.111, C.122, C. 123 , C. 138 , C.140, C.146, B.149, C. 160 .

PEIXATERIA, Plaça de la. (Límit de la Vilanova), C.139. PENAFREYTA. V. PRENAFETA.

PES DE LA FARINA, carrer del. (Límit de la Carnisseria). C.4. 
PICAMOIXONS. (Propietat i límit de la Magdalena i del Vergós). B.10, B.85, B.93, B.103, B.104, B.105, B.106, B.107, B.108, B.143, B.144, B.166.

PLA DE SANTA MARIA. (Límit de Palau de Reig i del Torrent del Pla). B.118, B.120, B.121, B.167.

PLA, Torrent del. (Limit de Palau de Reig). B.167.

POBLA, La. (Límit de l'Espinavesa i del Camí Moliner de la Granja de Dol de Llops). 145, B.164.

PONT DE GOI. (Propietat). B.151.

PONT DE NA PELLICERA. (Propietat). B.61.

PORTAL NOU, carrer del, (o carrer de la Carnisseria). C.42.

PRENAFETA. (Límit de Palau de Reig). B.129.

PRESÓ. (Límit de la Vilanova). C.29.

SABATERIA, Carrer de la. C.1, C.6, C.100, C.101, C.125, C.147.

SANTA ANNA, Església de. (Límit de la Vilanova). C.109.

SANTS, Coll dels. (Propietat). B.43, B.44.

SIMO, carrer d'en. C.34, C.87, C.95, C.96.

TARRAGONA. (Limit de Pont de Goi). C.7, B.151.

TELLARS, Quadra dels. (Limit dels Fontanals). B.55.

VALLMOLL. (Límit de l'Espinavesa i dels Fontanals). B.55, B.117.

VERGÓS. (Partida). B.9, B.58, B.162, B.166.

VERNEDA, La. (Partida). B.89, B.116, C.127.

VILAFRANCA, Torrent de la. (Límit de Palau de Reig). C.113, B.128, B.129, B.141, B.142.

VILANOVA, Camp de la. (Limit de la Bassa de la Bona). B.31. VILANOVA, Cap de la. (Propietat). B.27.

VILANOVA, carrer de la. C.3, C.12, C.14, C.15, C.22, C. 23 , C.29, C. 30 , C. 33 , C. 47 , C. 49 , C. 50 , C. 52 , C. 56 , C. 60 , C. 63 , C. 64 , C. 65 , C. 67 , C. 68 , C. 69 , C. 70 , C. 71 , C. 72 , C. 73 , C. 77 , C. 84 , C. 91 , C. 97 , C.99, C.102, C.109, C.112, C.124, C.132, C.136, C.139, C.150, C.165.

VILAR, Rec del. (Limit de l'Espinavesa i dels Fontanals). B.74, B.75, B.134.

VIOVER, Rec del. (Límit dels Fontanals). B.54. 